\title{
Precisão do conhecimento de resultados no processo adaptativo em crianças
}

CDD. 20.ed. 152.3

\author{
Rafael dos Santos HENRIQUE* \\ Aline Kety Maria de SIQUEIRA* \\ Natália Barros BELTRÃO* \\ Amanda Polyane Porto EPIFANIO* \\ Maria Teresa CATTUZZO"
}

${ }^{*}$ Escola Superior de Educação Física, Universidade de Pernambuco.

\section{Resumo}

0 objetivo deste estudo foi analisar os efeitos da precisão de Conhecimento de Resultados (CR) na aquisição de uma habilidade motora. Quarenta e oito crianças com idade entre oito e 10 anos ( $\widehat{\sigma}=9,33 \pm 0,70$ anos e $q=9,16 \pm 0,81$ anos) praticaram uma tarefa de acertar um alvo sobre uma mesa, mediante o lançamento de discos metálicos. Foram formados três grupos experimentais de acordo com o nível de precisão do CR: pouco preciso (GPP), preciso (GP) e muito preciso (GMP), além do grupo controle (GC), sem CR. A medida de desempenho foi o erro absoluto (EA). Os resultados indicaram diferença estatística a favor do GMP em comparação aos demais grupos, indicando que a maior precisão do CR favoreceu a estabilização da estrutura e a adaptabilidade do sistema, numa tarefa com meta de precisão espacial, em crianças.

Unitermos: Aprendizagem; Conhecimento de resultados; Prática; Desempenho psicomotor.

\section{Introdução}

Uma das principais características do início da aquisição de uma habilidade motora são os erros: o aprendiz, em face de inúmeras solicitações provenientes da tarefa, do ambiente e do controle do próprio corpo, nem sempre consegue responder com sucesso, de forma que, inicialmente, as ações são desorganizadas e pouco eficazes. Prática e a informação sobre o erro ("feedback") são as principais variáveis que modulam a aprendizagem motora (SALMONI, SChmidt \& Walter, 1984; Schmidt \& Lee, 1999).

De acordo com o Processo Adaptativo (TANI, 2005a; Tani, Bastos, Castro, Jesus, Sacay \& Passos, 1992), na aprendizagem de uma ação tanto a prática quanto o "feedback" levam à formação de uma estrutura mental abstrata, representada centralmente (Sistema Nervoso Central). Segundo TANI (2005b) essa representação está hierarquicamente arranjada em níveis: $1^{\circ}$ ) unidades de ação e algumas conexões estáveis entre elas; $2^{\circ}$ ) programas de ação, ou seja, a representação da sequência das unidades; e no $3^{\circ}$ nível, considerado o mais elevado e abstrato da hierarquia, a meta da ação é representada. Supõe-se que a organização de ações motoras envolve a ativação sucessiva desses três níveis de representaçãa. $\mathrm{O}$ contínuo processo de aprendizagem e desenvolvimento permite o aprimoramento dessas representações.

A formação da estrutura expressa-se mediante a padronização espaço-temporal da ação (estabilização do desempenho), e é o primeiro passo para o processo de aprendizagem concretizar-se. Depois disso, se uma perturbação é inserida nesta estrutura ora estável, incerteza e desordem são novamente geradas, que tendem a provocar alteraçôes. Ao ser perturbado o sistema pode ganhar em competência, pois para manter-se como tal ele precisa reorganizar-se, ganhando em complexidade.

Apesar de ser um teste à sua adaptabilidade, a perturbação não é o único evento que indica a capacidade de evolução do sistema: ele deve encontrar-se em um certo nível de organização, no qual a perturbação possa elicitar alguma resposta adaptativa (Tani, Correa, Benda \& Manoel, 2005). Assim, as características da prática e/ou "feedback" que modulam a formação e organização da estrutura, influenciam as respostas às perturbações, ou seja, o processo adaptativo em aprendizagem motora. 
O "feedback" aumentado sobre o resultado de uma ação, denominado de conhecimento de resultados (CR), é o dado externo que suplementa a fonte interna de informação sobre o erro na avaliação da ação ("feedback intrínseco") (ADAMs, 1987; SChmidt \& Lee, 1999). O CR auxilia o aprendiz a reduzir o desvio entre o que foi planejado e o que foi executado (TANI, 1989) e, assim como a prática, o CR poderia ser considerado fonte de ordem para o sistema (CATTUZZO \& TANI, 2009).

Uma das propriedades do CR éo seu grau de precisão (Mendes \& Godinho, 1994). Trowbridge e CASON (1932) citados por ADAMS(1987) podem ser considerados precursores da investigação sobre os efeitos da precisão do $\mathrm{CR}$ ao demonstrarem que CR mais específico (quantitativo) produzia aprendizagem mais rápida do que $\mathrm{CR}$ mais genérico (qualitativo). Alguns estudos têm evidenciado que um maior grau de precisão de CR tem efeito positivo no processo de aprendizagem (GILL, 1975; Godinho \& Mendes, 1996; Magill \& WoOd, 1986; Wright, Smith-MunYon \& SidaWAY, 1997). Conforme proposto pela hipótese de guia (SALMONI, SCHMIDT \& WALTER, 1984) a razão da influência positiva do aumento da precisão de CR parece estar ligada ao fato de que a informação mais exata sobre o erro permite que o aprendiz elabore os pontos necessários à modificação do comportamento. Porém, outros estudos não sustentaram estes achados (Jensen, Picado \& Morenz, 1981; Thomas, Mitchell \& Solmon, 1979).

Sob a ótica da abordagem teórica do Processo Adaptativo, dois estudos sobre precisão de CR foram realizados. TANI, MeIRA JúNIOR e GOMES (2005) investigaram o efeito do CR na aquisição de uma habilidade motora de preensão manual considerando as variáveis de precisão, frequência e localização temporal. Foram utilizados três tipos de precisão de CR: qualitativo, quantitativo, e qualitativo + quantitativo. Os resultados levaram os autores à conclusão de que a diminuição da frequência, das atividades interpoladas e da precisão do CR podem ser fontes de instabilidade que não prejudicaram a aprendizagem da tarefa. No entanto, estes três tipos de CR não necessariamente evidenciaram três níveis crescentes de precisão: o conhecimento isolado da direção do erro ou da distância do desvio (em $\mathrm{cm}$ ) podem equiparar-se em termos da especificidade do CR; assim somente existiu outro maior nível de precisão de CR quando era comunicado, concomitantemente, a direção e a distância do desvio do alvo.

Nesta mesma abordagem teórica, num estudo sobre a aprendizagem de uma tarefa com demandas de força e precisão espacial, MEIRA JúNIOR (2005) utilizou dois níveis de precisão de $\mathrm{CR}$ (geral e específico) em três diferentes frequências de CR (33\%, 66\% e 100\%). De acordo com o autor, a redução excessiva de incerteza, manipulada por intermédio de regimes de conhecimento de resultados mais precisos e frequentes, não se mostrou necessária pra o processo adaptativo em aprendizagem motora. Em suma, embora ambos os estudos evidenciem que menor grau de precisão do CR pareceu fornecer melhores condiçōes para a aprendizagem, ainda não é claro se existe um nível ótimo de precisão de $\mathrm{CR}$, tanto na formação da estrutura quanto na sua capacidade de adaptabilidade. Para isso seria necessário testar níveis crescentes dessa variável.

Considerando o exposto, o objetivo do presente estudo foi investigar o efeito de três níveis crescentes de precisão de CR na aprendizagem de uma habilidade motora, em crianças.

\section{Métodos}

\section{Amostra}

Esse é um estudo do tipo quase-experimental (Thomas \& Nelson, 2002) no qual 48 crianças (24 meninos, idade $=9,33 \pm 0,70$ e 24 meninas, idade $=9,16 \pm 0,81)$ de um projeto de extensão comunitária da ESEF - UPE de Recife participaram de forma voluntária. Os participantes não tinham experiência com a tarefa e tiveram o Termo de Consentimento Livre e Esclarecido assinado pelos responsáveis. $\mathrm{O}$ estudo contou com a aprovação do Comitê de Ética da UPE (protocolo no 42/08; CAAE 0042.0.097.000-08).

\section{Tarefa}

Os participantes praticaram uma tarefa de lançar discos metálicos em um alvo disposto horizontalmente sobre uma mesa, com o objetivo de acertar o centro. Uma cortina foi usada para impedir que o participante visse o seu desempenho e assim o CR verbal, dado pelo experimentador, era a única informação suplementar que o participante teria para desenvolver seu plano de ação. A escolha da tarefa justifica-se por: a) envolver meta clara; b) ser passível de execução e aprendizagem pelos participantes da pesquisa; c) possibilitar o fornecimento de CR de acordo com 
as manipulações previstas; e d) ser um tipo de tarefa muito utilizada na área de aprendizagem motora.

$\mathrm{O}$ alvo circular, pintado em tecido sobre uma mesa retangular (FIGURA 1), estava a uma distância de $2 \mathrm{~m}$ da borda da mesa que era cercada por uma borda de madeira de $20 \mathrm{~cm}$ de altura, forrada com material emborrachado; as

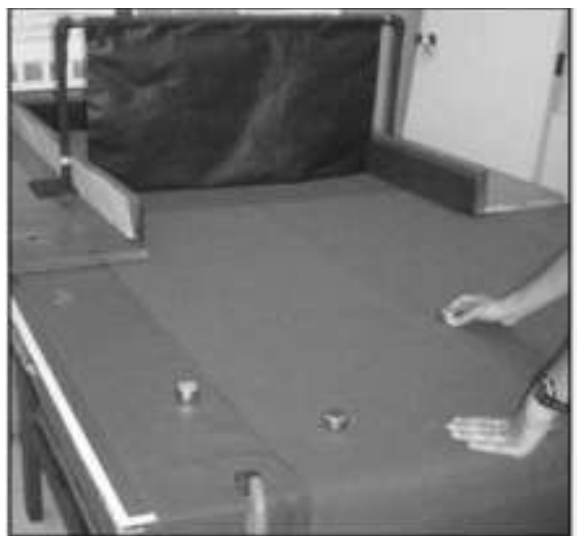

(a)

FIGURA 1 - Dispositivo experimental.

\section{Procedimentosedelineamentoexperimental}

A tarefa foi executada a partir da posição em pé, utilizando a mão dominante. Todos os grupos experimentais recebiam o CR verbal com $66 \%$ de frequência (duas tentativas consecutivas com CR, seguida por uma tentativa sem CR) com intervalo intertentativas de 10 segundos. Os sujeitos recebiam as instruçōes sobre a meta e as regras da tarefa, e em seguida executavam cinco tentativas-ensaio com visão total do alvo. Após o ensaio, a cortina era colocada para impedir a visão do alvo (FIGURA 1a). A coleta dos dados foi realizada numa das salas do Centro de Estudos da ESEF-UPE.

Os participantes foram distribuídos em quatro grupos de 12 sujeitos, equiparados em relação ao gênero. Os níveis de precisão da informação sobre o CR foram: no grupo pouco preciso (GPP) o CR indicava a cor da faixa do alvo; no grupo preciso (GP) o CR indicava a cor da faixa e o quadrante; no dimensóes dos discos metálicos foram 3,5 cm de diâmetro, $2 \mathrm{~cm}$ de altura e cada disco pesava $160 \mathrm{~g}$. Cada faixa do alvo media $7 \mathrm{~cm}$ de largura e a tarefa foi baseada no erro espacial da localização do disco. Assim, a cada faixa era atribuída uma pontuação. $\mathrm{O}$ alvo também foi dividido em quatro partes (Quadrantes 1, 2, 3 e 4) (FIGURA 1b).

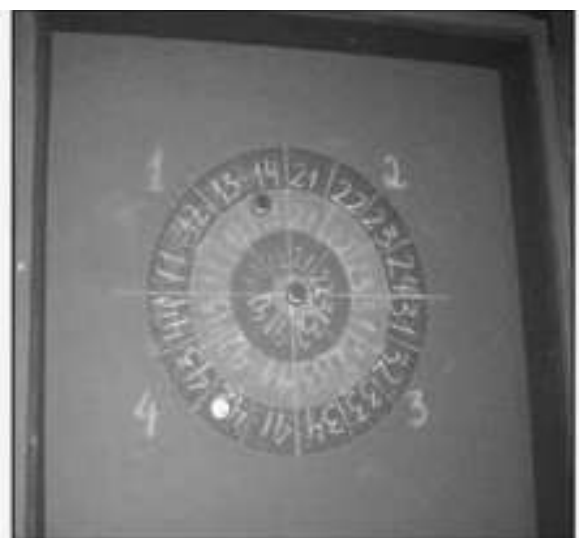

(b)

grupo muito preciso (GMP) o CR indicava a posição exata da localização do disco dentro do alvo que foi dividido em zonas numeradas (FIGURA 1b); o grupo controle (GC) não recebia CR (QUADRO 1).

O experimento teve duas fases: a estabilização com 60 tentativas e a adaptação com 10 tentativas. A quantidade de tentativas da fase de estabilização foi definida mediante estudo piloto. Logo ao término dessa fase, iniciava-se a de adaptação, com a introdução de uma perturbação caracterizada por um obstáculo - disco metálico semelhante ao do jogo, mas com $5 \mathrm{~cm}$ de diâmetro - posicionado antes da cortina, na direção do centro do alvo. Nessa fase os participantes não recebiam CR (QUADRO 1).

Uma vez que a meta da tarefa e, portanto, de aprendizagem, foi de precisão, a medida utilizada como variável dependente foi o erro absoluto (EA). Essa medida também foi usada em estudos anteriores sobre CR na abordagem teórica do Processo Adaptativo (TAni, Meira Júnior \& Gomes, 2005). $\mathrm{a}=$ mesa com a cortina que impedia a visão do alvo;

$\mathrm{b}=$ alvo circular com as marcações dos quatro quadrantes e as zonas numeradas de cada quadrante.

QUADRO 1 - Delineamento experimental discriminando os níveis de precisão de Conhecimento de Resultado (CR) de cada grupo, quantidade de prática nas fases de Estabilização e Adaptação e descrição da perturbação.

\begin{tabular}{|c|c|c|c|c|}
\hline $\mathbf{N}$ & Grupos & Fase de Estabilização & Perturbação & Fase de Adaptação \\
\hline 12 & $\begin{array}{c}\text { GC - Controle } \\
\text { (sem CR) }\end{array}$ & 60 tentativas & \multirow{2}{*}{$\begin{array}{c}\text { Colocação de um } \\
\text { obstáculo à frente do } \\
\text { centro do alvo, } \\
\text { antes da cortina }\end{array}$} & 10 tentativas \\
\cline { 1 - 2 } 12 & $\begin{array}{c}\text { GPP - Pouco Preciso } \\
\text { (CR da faixa do alvo) }\end{array}$ & 60 tentativas & 10 tentativas \\
\cline { 1 - 2 } 12 & $\begin{array}{c}\text { GP - Preciso } \\
\text { (CR da faixa do alvo e quadrante) }\end{array}$ & 60 tentativas & & 10 tentativas \\
\hline
\end{tabular}




\section{Análise dos dados}

Os escores do EA caracterizaram-se como variáveis numéricas discretas e os valores possíveis eram: $0,7,14$, 21 e 28 . O valor zero representava a meta, e o valor 28 representava fora do alvo. O desempenho do EA foi analisado mediante a somatória dos escores em blocos de cinco tentativas. Após a inspeção dos histogramas, verificação da normalidade da distribuição dos dados (teste Shapiro-Wilk) e homogeneidade da variância (teste de Levene) e, tendo sido atendidos os pressupostos para os testes paramétricos, foi realizada Análise de Variância (ANOVA) Two-Way para medidas repetidas tendo como fator independente o grupo e como medida repetida os blocos $(\mathrm{p} \leq 0,05)$; para contrastes posteriores foi usado o teste de Newman-Keuls. O nível de significância adotado em todas as análises foi de $\mathrm{p}<0,05$. Os pacotes estatísticos utilizados foram o SPSS 10.0 e Statistica 5.1.

\section{Resultados}

\section{Fase de estabilização}

A análise dos dados durante a fase de estabilização (FIGURA 2) indicou que o GMP obteve menor erro em comparação ao GC, GPP e GP (efeito do grupo: F3,44 $=8,70 ; \mathrm{p}<0,001$ ). Além disso, em todos os grupos, foi observado menor erro nos blocos finais em comparação aos blocos iniciais (efeito do bloco: F11,484 = 4,35; p < 0,001). Todavia, a análise da interação grupo*bloco não indicou efeito significante (efeito da interação: $\mathrm{F} 33,484=1,29 ; \mathrm{p}=0,130$ ).

\section{Fase de adaptação}

A análise dos dados durante a fase de adaptação (FIGURA 2) indicou que o GMP obteve menor erro absoluto em comparação ao GC, GPP e GP. Além disso, o GPP apresentou menor erro absoluto em comparação ao GC (efeito do grupo: F3,44 $=11,71 ; \mathrm{p}<0,001)$. Não foi observado efeito significante dos blocos $(\mathrm{p}>0,05)$ ou da interação $(\mathrm{p}>0,05)$.

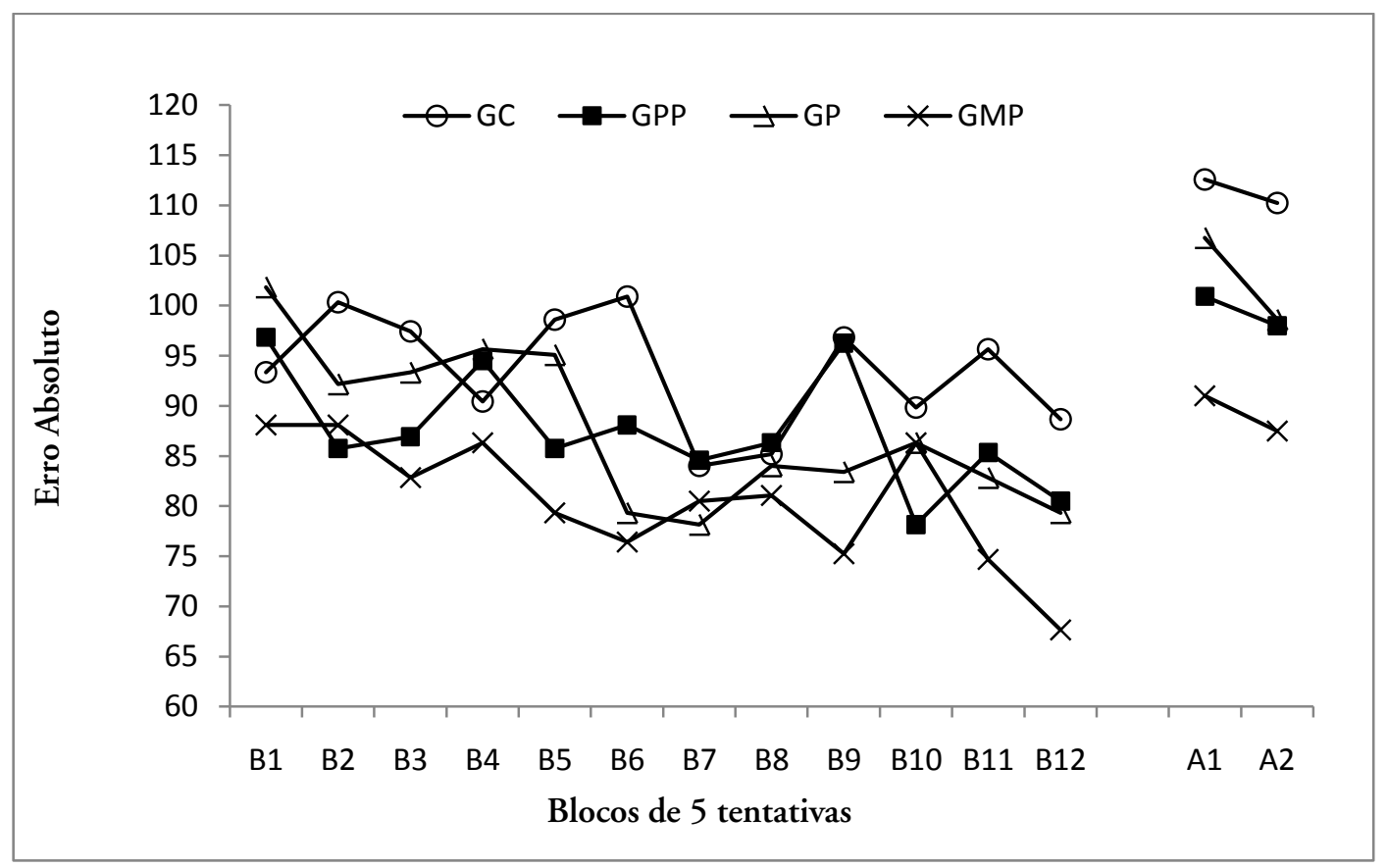

FIGURA 2 - Somatório do Erro Absoluto nas fases de Estabilização (B1 até B12) e de Adaptação (A1 e A2) do Grupo Controle (GC), Grupo Pouco Preciso (GPP), Grupo Preciso (GP) e Grupo Muito Preciso (GMP). 


\section{Discussão}

O objetivo do presente estudo foi investigar o efeito da precisão de CR na aprendizagem de uma tarefa manipulativa com meta de precisão espacial. Considerando que para a aprendizagem ocorrer deve haver incerteza presente no sistema, mas que ela seja passível de ser reduzida (GuAdaGnOlli \& LeE, 2004), esperava-se que regimes de CR muito específicos (GMP) ou muito genéricos (GPP) pudessem não surtir efeito positivo na aprendizagem. Além disso, em dois estudos na abordagem do processo adaptativo (MEIRA JÚNIOR, 2005; TANI, MEIRA JúNIOR \& GOMES, 2005) a maior precisão de CR pareceu não ser necessária para esse processo.

De acordo com os resultados, o GMP apresentou o melhor desempenho na estabilização e na adaptação em relação aos demais grupos, no entanto destaca-se que não houve efeito de interação, dificultando a localização da diferença entre os grupos em cada bloco. Pode-se observar que essa suposta superioridade do grupo que recebeu CR mais preciso assemelha-se aos achados de outras investigações. Por exemplo, no estudo de SHAPIRO (1977) crianças que receberam informação mais precisa sobre o erro obtiveram as melhores respostas em uma tarefa de posicionamento linear. Também SALMONI (1980), que investigou uma tarefa de desenho de linhas, confirmou estes resultados com crianças e adultos. Por outro lado, os resultados da presente investigação contrapõem-se aos de outros estudos os quais não confirmaram o efeito positivo do aumento da precisão de CR (LiTow \& LeVINe, 1978; Thomas, Mitchell \& Solmon, 1979).

Tratando de investigaçóes sob a abordagem do Processo Adaptativo, no desenho experimental de MeIra JúnIOR (2005) só foram utilizados dois níveis de precisão de CR, e no de Tani, Meira Júnior e GOMEs (2005) os três tipos de precisão utilizados não representaram, necessariamente, três níveis crescentes de precisão de CR: o conhecimento isolado da direção do erro (CR qualitativo) ou da distância do desvio (quantitativo) apesar de serem de naturezas distintas, continha o mesmo potencial de informação sobre o erro (em termos da incerteza presente na mensagem). Em ambos os estudos concluiu-se que menor precisão do $\mathrm{CR}$ pode ser fonte de instabilidade que não prejudica a aprendizagem da tarefa.

O desenho experimental do presente estudo contou com aumento crescente da precisão dessa variável em três níveis: a quantidade de informação sobre o erro (redução de incerteza) era gradativamente maior nos grupos, possibilitando a comparação de regimes extremos e intermediários de precisão de $\mathrm{CR}$. O conjunto dos resultados sugere que a maior exatidão da informação sobre o erro parece ter sido positiva para as crianças na aprendizagem dessa tarefa. Uma explicação para esse efeito encontra respaldo na hipótese de guia do CR (Salmoni, Schmidt \& Walter, 1984), no sentido que a informação mais precisa sobre o erro pode ser a orientação que melhor direciona o processamento da informação.

A hipótese de guia do CR está formatada numa visão de aprendizagem na qual o conhecimento sobre o erro constitui-se na informação que irá fortalecer o esquema na sua operação de especificar respostas motoras e suas correçôes. No entanto, numa outra abordagem para compreender e avaliar a aprendizagem, o conhecimento sobre o erro pode ser pensado como mais um elemento que combate a entropia a qual todos os sistemas abertos estão sujeitos, importando ordem para o sistema. Assim os erros podem ser vistos como flutuações necessárias na formação de um estado estável (MANOEL, 2001). Poder-se-ia especular que a padronização da estrutura espaço-temporal da ação (estabilização do desempenho) alcançada com o CR muito preciso levou o sistema para um estado estável que permitiu suportar melhor a perturbação.

A aparente vantagem que as crianças obtiveram com a estabilização do desempenho alcançada com CR muito preciso poderia ser um exemplo de ordem gerando ordem. O conhecimento específico e minucioso sobre o erro pode ser entendido como uma informação com pouca ou nenhuma incerteza. Para as crianças, imersas em incertezas sobre si mesma, sobre a tarefa e sobre o ambiente, isso parece ter sido vantajoso. Embora só possamos nos basear nos dados descritivos, é notável que a partir dos segundo bloco de prática os grupos que receberam CR tenderam a diminuir seus erros, mas o GMP destacou-se com desempenho superior. Para as crianças do GMP, maior precisão do CR pode ser fonte de estabilidade que beneficiou a aprendizagem da tarefa.

Assim, essa estrutura parece ter sido formada com a estabilidade necessária para fazer frente à perturbação, fator gerador de instabilidade no sistema. Pensando a aprendizagem como um processo contínuo que depende da participação direta tanto da instabilidade quanto da estabilidade, já que são momentos diferentes ("ordem através da desordem" para criar uma estrutura e "ordem através da ordem" para a sua continuidade) parece, então, haver ciclos de instabilidade-estabilidade-instabilidade que levam o sistema para níveis de complexidade superiores (CATTUZZO, 2007; TANI, 2005a). 
Ademais, os achados da presente investigação precisam considerar que o nível de desenvolvimento pode ser uma variável interveniente nesse processo. Crianças podem beneficiar-se de informações mais precisas sobre seu desempenho, pois isso auxilia o foco atencional e o maior envolvimento cognitivo com a tarefa a ser aprendida. Os estudos na abordagem do Processo Adaptativo sobre precisão do CR foram realizados com adultos. Considerando a aprendizagem como um processo contínuo e não-linear e de aumento de complexidade (TANI, 2005a), a quantidade de experiências acumulada pelos adultos - maior base de conhecimento e complexidade - pode ser suficiente para prover as correçôes necessárias durante a prática, sem que eles tenham que recorrer ao conhecimento específico sobre o erro, como as crianças o fizeram. No entanto estas especulações poderiam ser testadas com crianças e adultos sendo submetidos à aprendizagem da mesma tarefa, nas mesmas condições.

Nossos resultados também precisam ser considerados com respeito à escala métrica utilizada para informar o $\mathrm{CR}$, pois pode existir problemas na capacidade de processar informação sobre o erro, ligado ao reconhecimento dessa escala (NEWELL \& Kennedy, 1978). A escala métrica externa (inerente ao dispositivo experimental ou à tarefa) pode não estabelecer relação com a escala métrica interna ao aprendiz (Fajen, Riley \& Turvey, 2008). No estudo de Tani, Meira Júnior e Gomes (2005), por exemplo, a escala métrica utilizada para o CR quantitativo da tarefa de preensão manual (escala de força) pode não ser uma informação comumente/naturalmente utilizada pelos indivíduos. Ela pode não ser relevante, e o aprendiz pode ter dificuldade de associá-la ao erro de sua ação. O dispositivo experimental utilizado no presente estudo continha uma escala que pode ser considerada ecologicamente válida, o que pode ter facilitado o processamento da precisão do CR.

Outra especulação originada da análise do presente estudo diz respeito ao uso efetivo do CR pelo aprendiz quando ele é dado pelo experimentador. Foi possível observar que, durante a coleta de dados, o arranjo de 66\% de frequência de CR externamente controlado muitas vezes não atendia à expectativa do sujeito em conhecer seu erro, ou ele era dado quando o sujeito não o desejava. Assim, apesar de ser considerada uma delimitação no presente estudo, a situação de controle do regime de CR (controle externo x auto-controle) poderia ser manipulada em outro experimento com essa mesma tarefa. É importante enfatizar que já há resultados na literatura confirmando os efeitos positivos do autocontrole de CR. Por exemplo, os resultados do estudo de Chiviacowsky, Wulf, Medeiros, KaEFER e TANI (2008) evidenciaram superioridade para o grupo autocontrolado, em relação ao "yoked", na fase de retenção com crianças quando a tarefa exigia precisão espacial e força.

Em suma, considerando o exposto, entendese que os resultados da presente investigação ao confirmarem o efeito positivo do aumento da precisão de CR em crianças, parecem complementar os resultados já encontrados sob a abordagem do Processo Adaptativo, uma vez que se pode esperar que o ser humano, como um sistema complexo, mude sua competência de adaptação ao longo dos ciclos instabilidade-estabilidade-instabilidade que caracterizam os processos de aprendizagem e desenvolvimento (CATTUZZo, 2007).

\title{
Conclusão
}

Os resultados do presente estudo permitem concluir que a maior precisão do CR favoreceu a formação da estrutura e sua adaptabilidade, numa tarefa com meta de precisão espacial, em crianças.

\begin{abstract}
Precision of knowledge of results in the adaptative process in children

This study aimed to analyze the effects of Knowledge of Results (KR) precision on acquisition of a motor ability. Forty eight children aged between eight and 10 years old $(\hat{\sigma}=9.33 \pm 0.70$ years old; $q=9.16 \pm 0.81$ years old) practiced a manipulative task of reaching a target on a table, by the thrown of metallic discs. Three experimental groups were formed according to the KR precision level: less precise group (LPG), precise group
\end{abstract}


(PG) and very precise group (VPG), and also the control group (CG), without KR. The performance measure was the absolute error. The results indicated statistic differences in favor of VPG when compared to the other groups, indicating that higher KR precision benefited the system's stabilization and adaptability for task with accuracy goal, for children.

UnITERMS: Learning; Knowledge of results; Practice; Psychomotor performance.

\section{Notas}

Rafael dos Santos Henrique é Bolsista PIBIC/CNPq/UPE Processo nº 112738/2008-7.

Aline Kety Maria de Siqueira é Bolsista PIBIC/CNPq/FACEPE.

Natália Barros Beltrão é Bolsista IC/PFAUPE/CNPq.

Amanda Polyane Porto Epifanio é Bolsista IC/PFAUPE/CNPq.

\section{Referências}

ADAMS, J.A. Historical review and appraisal of research on the learning, retention, and transfer of human motor skills. Psychological Bulletin, Lancaster, v.101, p.41-74, 1987.

CATTUZZO, M.T. O ciclo instabilidade-estabilidade no processo adaptativo em aprendizagem motora. 2007. Tese (Doutorado) - Escola de Educação Física e Esporte, Universidade de São Paulo, São Paulo, 2007

CATTUZZO, M.T.; TANI, G. Estudo da aprendizagem motora: uma pequena síntese histórica e algumas perspectivas para o seu desenvolvimento. In: CATTUZZO, M.T.; TANI, G. Leituras em biodinâmica e comportamento motor: conceitos e aplicações. Recife: EDUE, 2009

CHIVIACOWSKY, S.; WULF, G.; MEDEIROS, F.L.; KAEFER, A.; TANI, G. Learning benefits of self-controlled of results in 10-year-old children. Research Quarterly for Exercise and Sport, Washington, v.79, n.3, p.405-10, 2008.

FAJEN, B.R.; RILEY, M.A.; TURVEY, M.T. Information, affordances, and the control of action in sport. Journal of Sport Psychology, Champaign, v.40, p.79-107, 2008.

GILL, D.L. Knowledge of results precision and motor skill acquisition. Journal of Motor Behavior, Washington, v.7 n.3, p.191-8, 1975.

GODINHO, M.; MENDES, R. Aprendizagem motora: informação de retorno sobre o resultado. Lisboa: FMH, 1996.

GUADAGNOLI, M.A; LEE, T.D. Challenge point: a framework for conceptualizing the effects of various practice conditions in motor learning. Journal of Motor Behavior, Washington, v. 36, n.2, p.221-24, 2004.

JENSEN, B.; PICADO, M.; MORENZ, C. Effects of precision of knowledge of results on performance of a gross motor coincidence-antecipation task. Journal of Motor Behavior, Washington, v.13, n.1, p.9-17, 1981.

LITOW, L.; LEVINE, S.M. Effects of training to use feedback and responsiveness to information on preschool children discrimination learning. Perceptual and Motor Skills, Missoula, v.47, p.547-64, 1978.

MAGILL, R.A.; WOOD, C.A. Knowledge of results precision as a learning variable in motor skill acquisition. Research Quarterly for Exercise and Sport, Washington, v.57, n.2, p.170-3, 1986.

MANOEL, E. O diálogo no processo de aquisição de habilidades motoras. In: GUEDES, M.G.S. (Ed.) Aprendizagem motora: problemas e contextos. Lisboa: Edições FMH, 2001.

MEIRA JÚNIOR, C.M. Conhecimento de resultados no processo adaptativo em aprendizagem motora. 2005. Tese (Doutorado) - Escola de Educação Física e Esporte, Universidade de São Paulo, São Paulo, 2005.

MENDES, R.; GODINHO, M. Knowledge of results precision and learning: a review. Revista de Psicologia del Deporte, Palma de Mallorca, v.6, p.23-34, 1994.

NEWELL, K.M.; KENNEDY, J.A. Knowledge of results and children's motor learning. Development Psychology, Washington, v.14, n.5, p.531-6, 1978.

SALMONI, A. The effect of precision of knowledge of results on the performance of a simple line drawing task for children and adults. Research Quarterly for Exercise and Sport, Washington, v.51, n.3, p.572-5, 1980. 
SALMONI, A.; SCHMIDT, R.A.; WALTER, C.B. Knowledge of results and motor learning: a review and critical reappraisal. Psychological Bulletin, Lancaster, v.95, p.355-86, 1984.

SHAPIRO, D. Knowledge of results and motor learning in preschool children. Research Quarterly for Exercise and Sport, Washington, v.48, n.1, p.154-8, 1977.

SCHMIDT, R. A.; LEE, T.D. Motor control and learning: a behavioral emphasis. 3rd. ed.Champaign: Human Kinetics, 1999. TANI, G. Significado, detecção e correção do erro de performance no processo ensino-aprendizagem de habilidades motoras. Revista Brasileira de Ciência e Movimento, Brasília, v.3, n.4, p.50-8, 1989.

Processo adaptativo: uma concepção de aprendizagem motora além da estabilização In: TANI, G. (Ed.) Comportamento motor: aprendizagem e desenvolvimento. São Paulo: Guanabara Koogan, 2005a.

Programação motora: organização hierárquica, ordem e desordem. In: TANI, G. (Ed.) Comportamento motor: aprendizagem e desenvolvimento. São Paulo: Guanabara Koogan, 2005b.

TANI, G.; BASTOS, F.C.; CASTRO, I.J.; JESUS, J.F.; SACAY, R.C.; PASSOS, S.C.E. Variabilidade de resposta e processo adaptativo em aprendizagem motora. Revista Paulista de Educação Física, São Paulo, v.6, p.16-25, 1992.

TANI, G.; CORREA, U.C.; BENDA, R.N.; MANOEL, E.J. O paradigma sistêmico e o estudo do comportamento motor humano. In: TANI, G. (Ed.) Comportamento motor: aprendizagem e desenvolvimento. São Paulo: Guanabara Koogan, 2005. TANI, G.; MEIRA JÚNIOR, C.M.; GOMES, F.R.F. Frequência, precisão e localização temporal de conhecimento de resultados e o processo adaptativo na aquisição de uma habilidade motora de controle de força manual. Revista Portuguesa de Ciências do Desporto, Porto, v.5, n.1, p.59-68, 2005.

THOMAS, J.; MITCHELL, B.; SOLMON, M. Precision knowledge of results and motor performance: relationship to age. Research Quarterly for Exercise and Sport, Washington, v.50, n.4, p.687-98, 1979.

THOMAS, J.R.; NELSON, J.K. Métodos de pesquisa em atividade física. 3.ed. Porto Alegre: Artmed, 2002.

WRIGHT, D.L.; SMITH-MUNYON, V.L.; SIDAWAY, B. How close is too close for precise knowledge of result? Research Quarterly for Exercise and Sport, Washington, v.68, n.2, p.172-6, 1997.

ENDEREÇO

Rafael dos Santos Henrique

R. Paes Cabral, 357 - apto. 7 50630-170 - Recife - PE - BRASIL e-mail: rdshenrique@hotmail.com
Recebido para publicação: 15/09/2009

1a. Revisão: 08/02/2010

Aceito: 18/02/2010 